\title{
Martian magnetic morphology: Contributions from the solar wind and crust
}

\author{
D. A. Brain and F. Bagenal \\ Laboratory for Atmospheric and Space Physics, University of Colorado, Boulder, Colorado, USA
}

\author{
M. H. Acuña and J. E. P. Connerney \\ NASA Goddard Space Flight Center, Greenbelt, Maryland, USA
}

Received 11 May 2002; revised 12 February 2003; accepted 23 May 2003; published 5 December 2003.

[1] Mars Global Surveyor (MGS) Magnetometer (MAG) data provide constraints on magnetic morphology at Mars, including the relative importance of the solar wind and of crustal magnetic sources. We analyze MAG data to characterize the upstream interplanetary magnetic field (IMF) and confirm trends in the magnetic field expected from the solar wind interaction with a planetary atmosphere, including increases at the shock and magnetic pile-up boundary (MPB), postshock turbulence, and field line draping around the Martian obstacle. Crustal magnetic sources locally modify the solar wind interaction, adding variability to the Martian magnetic environment that depends on planetary rotation. We identify trends in the vector magnetic field with respect to altitude, solar zenith angle, and planetary location. Crustal sources influence the magnetic field to different altitudes above different regions, and the influence of the strongest source extends to $1300-1400 \mathrm{~km}$. The draped IMF partially controls the field topology above crustal sources, and crustal magnetic field lines reconnect to this field in a systematic fashion that depends upon Mars' geography, IMF strength, and IMF orientation. INDEX TERMS: 6225 Planetology: Solar System Objects: Mars; 5440 Planetology: Solid Surface Planets: Magnetic fields and magnetism; 5421 Planetology: Solid Surface Planets: Interactions with particles and fields; 5430 Planetology: Solid Surface Planets: Interiors (8147); KEYWORDS: Mars, crustal magnetism, solar wind interaction, magnetic field topology, Mars Global Surveyor

Citation: Brain, D. A., F. Bagenal, M. H. Acuña, and J. E. P. Connerney, Martian magnetic morphology: Contributions from the solar wind and crust, J. Geophys. Res., 108(A12), 1424, doi:10.1029/2002JA009482, 2003.

\section{Introduction}

[2] Both the solar wind and the crust influence magnetic field morphology near Mars [Acuña et al., 1998]. The influence of the solar wind has been studied for decades, after early spacecraft to Mars showed that the intrinsic global magnetic field, if any existed at all, was weak (see review by Luhmann et al. [1992]). Models for the interaction focused on the ionosphere as the obstacle to the solar wind, similar to the interaction at Venus and at comets [Spreiter and Stahara, 1980; Brecht, 1997; Liu et al., 1999]. In this interaction the solar wind slows postshock, interacts with exospheric neutrals and ions, and diverts around the planet. Magnetic field lines entrained in the plasma flow "drape" around the planetary obstacle. The structure of the magnetic field near Mars in this interaction has several observable features: an increase in field magnitude and turbulence below the shock, nearly horizontal field vectors with respect to the planetary surface on the dayside, a rotation in magnetic field below the ionopause, and a tail region with two lobes of opposite magnetic polarity (con-

Copyright 2003 by the American Geophysical Union. 0148-0227/03/2002JA009482\$09.00 trolled by that of the upstream interplanetary magnetic field). Early spacecraft to Mars observed many of these features [Vaisberg, 1992; Zakharov, 1992, and references therein].

[3] More recently, the MGS magnetometer and electron reflectometer (MAG/ER) confirmed that any existing Martian magnetic dipole moment must be very small (reducing previous estimates of the upper limit by a factor of 5-10), and demonstrated that the crust contains regions of strongly magnetized material [Acuña et al., 1998]. The strongest crustal sources are located in the Terra Sirenum region of Mars. Other strong sources are located on the ancient cratered highlands near the Martian dichotomy boundary and weaker sources are observed in the north [Acuña et al., 1999]. Acuña et al. [1999] estimated the net magnetic moment of $1.3 \times 10^{17} \mathrm{~A}-\mathrm{m}^{2}$ for the strongest crustal sources. The measured field strength from this source at $400 \mathrm{~km}$ altitudes exceeds the field strength at similar altitudes above the largest magnetic anomalies on Earth by a factor of 20 [Connerney et al., 1999].

[4] The strength of crustal sources, combined with the lack of a global Martian magnetic field, suggests that crustal magnetic field sources affect the Martian solar wind interaction to high altitudes. This interaction has interesting 
implications. Crustal sources contribute magnetic pressure $\left(B^{2} / 2 \mu_{0}\right)$ that can offset the solar wind dynamic pressure $\left(\rho v^{2}\right)$ above certain regions on the surface, resulting in a Martian solar wind obstacle with an irregular shape. As the planet rotates and different regions of the obstacle are exposed to the solar wind the obstacle exhibits inherent time variability. Furthermore, reconnection of crustal magnetic field lines to interplanetary magnetic field lines is not considered in any existing model for the Martian magnetic environment, despite its implications for charged particle motion and acceleration near Mars. The issues of atmospheric loss, particle deposition in the upper atmosphere, and surface radiation are all affected by the non-uniformity of the Martian solar wind obstacle and the topology of the magnetic field near crustal sources.

[5] MGS MAG/ER data provide information about the relative contributions of the solar wind and crust to magnetic field observations near Mars. We present an analysis of Martian magnetic field morphology as observed by the MAG/ER magnetometer. We summarize gross magnetic features of the solar wind interaction with Mars from outside of the shock to the planetary nightside and of the crustal sources. We then explore the combined influence of crustal and interplanetary magnetic fields, taking into account parameters associated with planetary rotation, longitude, latitude, altitude, and solar zenith angle (SZA). Our specific emphasis is on the unique aspects of the magnetic field morphology that result from the interaction of the solar wind with crustal magnetic sources.

\section{Data Coverage}

[6] The MGS MAG/ER investigation and data set are described by Acuña et al. [1992], Acuña et al. [1999], and Acuña et al. [2001]. The information obtained by MAG about the crust and the solar wind interaction depends largely upon the spacecraft orbit. The MGS mission can be divided into two distinct phases: premapping and mapping.

[7] The term "premapping" refers collectively to data from four MGS mission phases during aerobraking. Premapping observations used in this work were recorded between 13 September 1997 and 28 January 1999. During this period the spacecraft orbit was elliptical, near-polar, and evolved in local time. Periapsis altitudes range from northern midlatitudes early in the mission to southern latitudes for the months prior to the commencement of mapping. Altitudes of periapsis ranged from $\sim 101 \mathrm{~km}$ to $\sim 160 \mathrm{~km}$, and the maximum apoapsis was $\sim 17 \mathrm{R}_{\mathrm{M}}$. Premapping data provide unprecedented coverage of the Martian solar wind interaction. The local time of the MGS orbit plane precessed approximately 16 hours from a dusk-dawn orientation to a $2 \mathrm{pm}-2 \mathrm{am}$ orbit during this time period. The ellipticity of the orbit decreased simultaneously, resulting in better coverage of the Martian magnetic environment in altitude and solar zenith angle than any previous spacecraft. Figure 1a shows data coverage for premapping orbits in Sun-state cylindrical coordinates (a right handed coordinate system with the $\mathrm{x}$-axis pointing toward the Sun and the y-axis pointing opposite to Mars' orbital motion). Most lowaltitude observations were made at solar zenith angles between $30^{\circ}$ and $150^{\circ}$, with many observations throughout the sheath and upstream of the Martian shock. Premapping
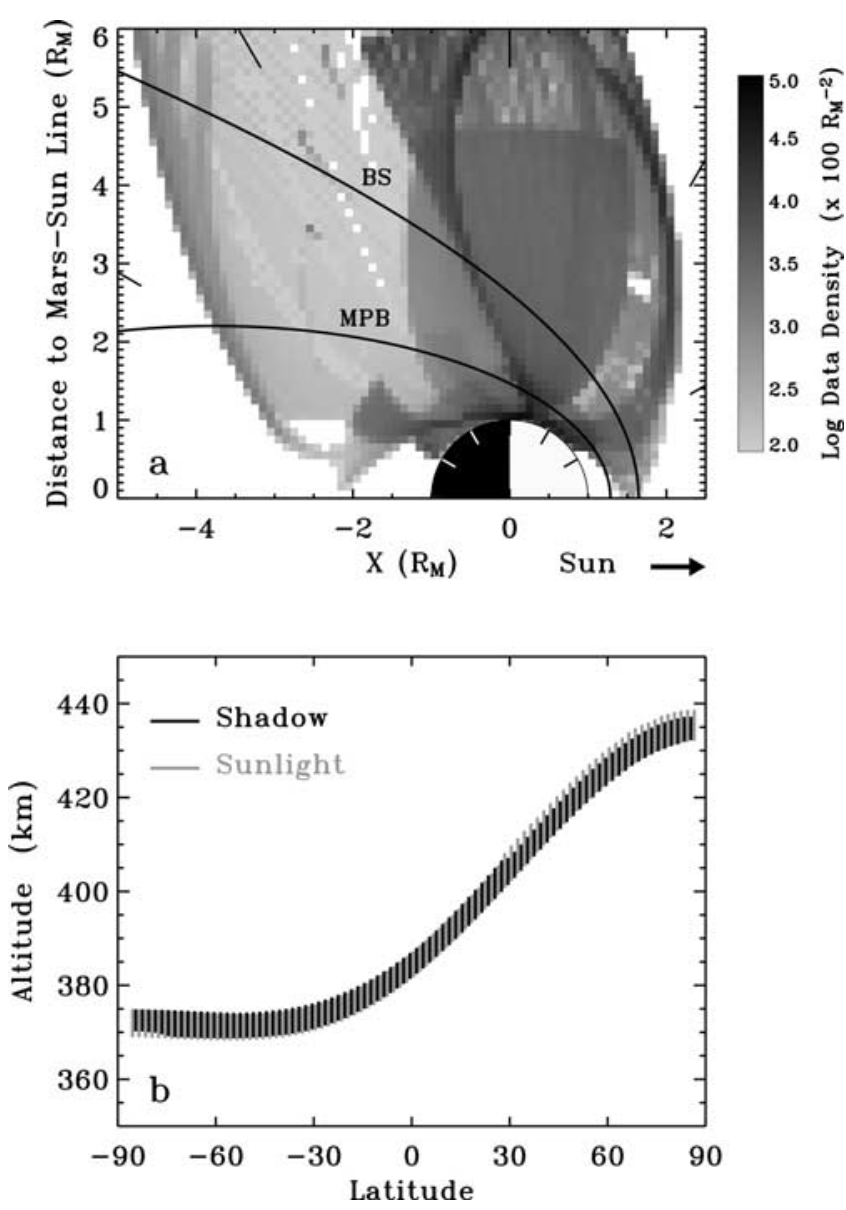

Figure 1. MGS MAG Data coverage. (a) Data density for premapping orbits in Sun-state cylindrical coordinates. The best fit shock (BS) and and MPB boundaries [Vignes et al., 2000] are shown as solid lines and labeled. Hash marks note solar zenith angle every $30^{\circ}$. (b) Data coverage as a function of latitude for mapping orbits, separated according to whether MGS was in shadow or sunlight. Within each latitude bin, the maximum and minimum observation altitudes are connected by a vertical bar.

data are also useful for obtaining important low-altitude observations of crustal sources but do not provide uniform coverage of the planetary surface at any altitude.

[8] MGS achieved a 2pm-2am mapping orbit in February of 1999. The shape of the spacecraft orbit varied for the first several weeks of mapping; therefore we use mapping data taken between 2 June 1999 and 31 January 2001 for this work. Mapping data are very useful for examining the crustal contribution to the magnetic environment at constant altitude [Connerney et al., 2001]. Figure 1b shows the altitude coverage as a function of latitude during mapping. The spacecraft periapsis for the nearly circular orbit occurs very close to the South Pole; as a result, the spacecraft altitude at a given latitude on the dayside of the planet is very similar to the altitude on the nightside. We assume that Mars is an ellipsoid with equatorial radius of $3397 \mathrm{~km}$ and polar radius of $3375 \mathrm{~km}$ when computing altitude. The longitude coverage at a given latitude is uniform over scales of one degree or more. Mapping observations provide limited information about the interplanetary magnetic field. 
No upstream solar wind monitor exists at Mars, and at $\sim 400 \mathrm{~km}$ altitudes reliably extracting external field contributions from the data for individual orbits proves difficult. However, multiple spacecraft passes over the same region of the surface can be used to determine the variability in magnetic field at the mapping altitude. Further information about MAG data coverage in solar zenith angle, altitude, local time, and latitude at low altitudes is available in the work of Brain [2002].

\section{Morphology of the Solar Wind Interaction}

[9] As Figure 1 demonstrates, the MGS premapping orbit geometry enabled magnetic field observations to be made from upstream of the shock to the Martian tail region. Prior to MGS the exact nature of the Martian obstacle to the solar wind was debated, with arguments for a magnetospheric obstacle [e.g., Dolginov et al., 1976], an ionospheric obstacle [e.g., Russell et al., 1978], a combined magnetospheric/ ionospheric obstacle [e.g., Bogdanov and Vaisberg, 1975], and even a combined (ionospheric/remanent magnetism) obstacle [Curtis and Ness, 1988]. The most convincing evidence supported either an ionospheric or combined obstacle, and early observations detected a number of characteristic boundaries and plasma regions near Mars with similarities to those observed at Venus and comets [see Breus, 1992]. On the planetary dayside three main boundaries have been described by MGS [Acuña et al., 1998]. A shock forms as a result of the solar wind interaction with the ionospheric obstacle and, to first order, marks the location where the solar wind begins to slow and divert around the planet. Downstream from the shock, the Magnetic Pile-up Boundary (MPB) (called the cometopause, planetopause, and Mass Loading Boundary at other bodies or by other instrument teams) separates plasma dominated by solar wind protons from plasma significantly affected by planetary ions [e.g., Breus et al., 1992]. At lower altitudes, the photoelectron boundary is identified mainly by a change in the energetic electron flux, with shocked solar wind electrons above the boundary and ionospheric photoelectrons below the boundary [Mitchell et al., 2001]. This boundary may be colocated with the ionopause, which is considered by many to be a physical boundary where planetary pressure contributions balance solar wind dynamic pressure, including ionospheric thermal pressure and magnetic pressure from ionospheric fields [Mitchell et al., 2001]. Between the photoelectron boundary and MPB, a region called the Magnetic Pile-up Region (also called the mantle or the ion cushion) is characterized by a sustained high magnetic field magnitude and the presence of heavy planetary ions [Szego, 2001]. The region between the MPB and the shock is called the sheath, the magnetosheath, or the ionosheath, but the term sheath also sometimes refers to the entire region between the shock and the ionopause. The tail region of Mars contains "draped" convected magnetic field lines, and some experiments have indicated that it is bounded by a turbulent boundary layer that is an extension of the Magnetic Pile-up Region populated by escaping low-energy ionospheric ions [Lundin et al., 1990a]. High heavy ion fluxes have been reported at the center of the tail region, consistent with a plasma sheet [Lundin et al., 1990b].
[10] As the spacecraft travels toward Mars from the region upstream of the bow shock, an increase in field magnitude and variability is expected after crossing the shock. Also, the magnetic field lines will begin to drape around the obstacle. At the MPB, the variability in the field will decrease, and the field vector will rotate and increase in magnitude. At the ionopause the field magnitude will not change significantly, but a rotation of the horizontal field component was observed at the Venusian ionopause [ Law and Cloutier, 1995]. In the tail, the draped magnetic field lines form a two-lobed structure: one portion of the tail region contains a magnetic field with a positive sunward (or $B_{\mathrm{x}}$ ) component, and the other portion contains a field with negative $B_{\mathrm{x}}$. These trends have been confirmed for individual MGS orbits or subsets of premapping data [Acuña et al., 1998; Cloutier et al., 1999; Vignes et al., 2000; Crider et al., 2001]. Here we describe basic aspects of the solar wind interaction with Mars using the entire MAG data set acquired during the MGS mission phases described in section 2 .

[11] Early in the premapping portion of the mission, the MGS orbit allowed characterization of the unperturbed IMF at 1.5 AU. Figure 2 illustrates the IMF at the orbit of Mars. A histogram of all premapping observations determined to be substantially upstream from the Martian shock shows that the field magnitude peaks between 2.5 and $3 \mathrm{nT}$. A spiral angle to the magnetic field with a median value of $52^{\circ}$ is evident, and the field vector points downstream more often than it points upstream (see the second panel of Figure 2). Additionally, a majority of magnetic field vectors lie within $30^{\circ}$ of the ecliptic plane. These observations agree with the results from the Mars 2, Mars 3, and Mars 5 spacecraft [Luhmann et al., 1992, and references therein] as well as from the Phobos 2 spacecraft [Schwingenschuh et al., 1990]. The field measurements presented here were recorded from late 1997 to early 1999, as solar activity increased from solar minimum toward solar maximum. We note that magnetic fields from the MGS spacecraft itself (removed to an accuracy of $\sim 1 \mathrm{nT}$ in sunlight [Acuña et al., 2001]) make reliable determination of magnetic field direction difficult, especially in regions where the field magnitude is weak.

[12] We examined the entire premapping data set to identify trends in field magnitude and variability. Figure $3 \mathrm{a}$ shows the median magnetic field magnitude as a function of spacecraft position in Sun-state cylindrical coordinates. The field magnitude increases at the shock at all solar zenith angles and again at the MPB on the dayside. The postshock field is greatest at low solar zenith angles for a given altitude. The strongest field magnitudes are observed closest to the planet, contrary to expectations from a simple solar wind interaction with an ionosphere, and are attributable to crustal magnetic sources. The RMS in field magnitude was calculated every $30 \mathrm{~s}$ from all premapping data. In order to calculate variation on $30 \mathrm{~s}$ timescales, the data were digitally resampled at a $3 \mathrm{~s}$ data rate (to remove any effects from changes in the instrumental sampling rate) and linear filtered (effectively finding the deviation in $\Delta B$ from one measurement to the next) to remove the effects of crustal sources, which contribute to RMS but change the measured field smoothly. Figure $3 \mathrm{~b}$ shows that the observations display the most variability between the bow shock and 


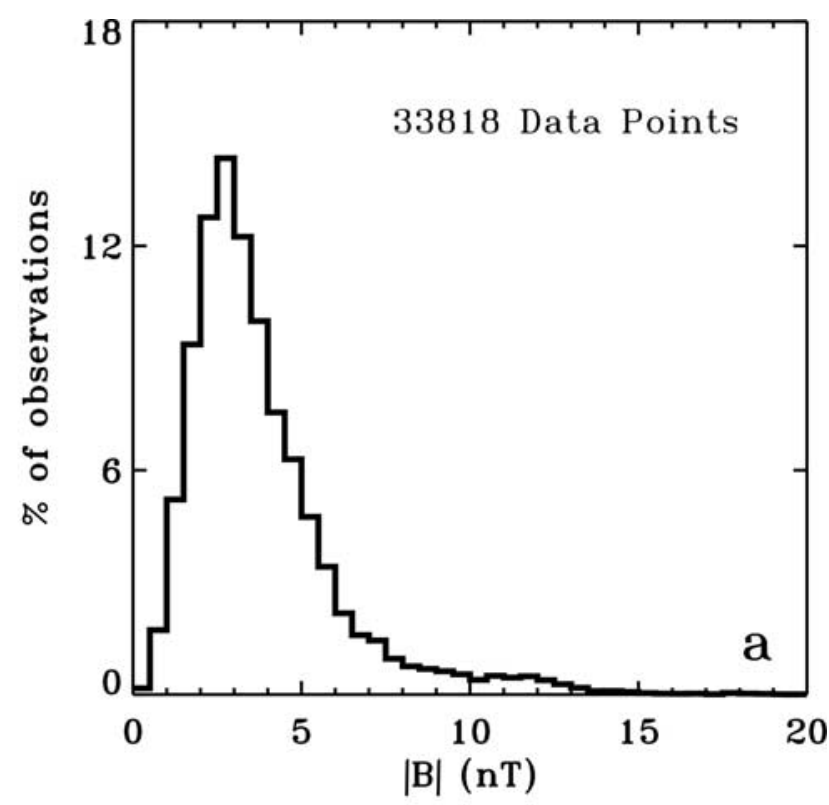

ionosphere using magnetometer data from MGS. We used premapping data in a manner similar to that for Figure 3 to illustrate the draping of the IMF throughout the Martian system. We again resampled the observations at a $3 \mathrm{~s}$ data rate and averaged every $30 \mathrm{~s}$ to remove high-frequency oscillations in the field. This process resulted in a data set with sampling interval of $30 \mathrm{~s}$ where each data point represented an average of 10 observations over those $30 \mathrm{~s}$. The median magnetic field vector in cubic bins measuring $0.25 \mathrm{R}_{\mathrm{M}}$ on a side was determined, rejecting bins with fewer than 25 observations. The orientation of the magnetic field near Mars depends upon the orientation of the upstream IMF; before finding the median we removed a sign ambiguity by reversing all vectors for which $B_{\mathrm{y}}$ was negative. The expected solar wind aberration near Mars is small (approximately $3.5^{\circ}$ ), so we expect the field draping picture to be approximately symmetric about the $\mathrm{x}$-axis. Figure 4 shows several aspects of the IMF draping. Upstream from the shock the magnetic field has the properties (small field magnitude, spiral angle) demonstrated in Figure 2. The

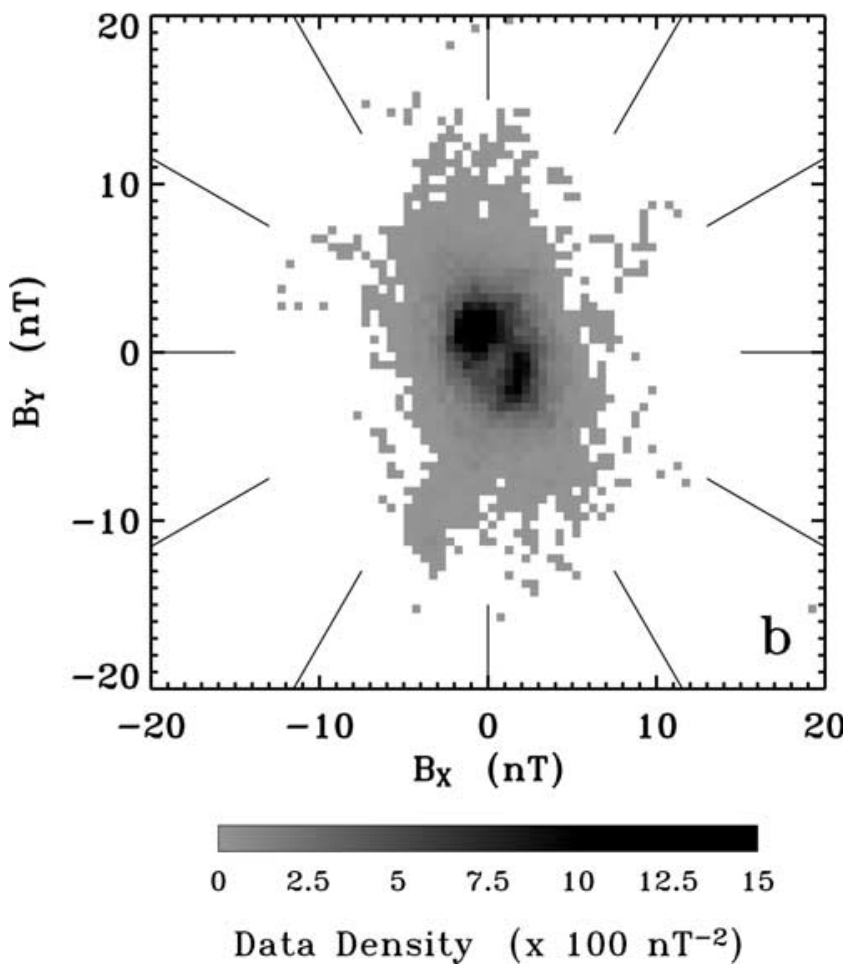

Figure 2. The unperturbed IMF at 1.5 AU. Data significantly upstream from the bow shock were averaged together in $5 \mathrm{~min}$ intervals. (a) Histogram of field magnitudes. (b) Data density of field vectors projected onto the $B_{\mathrm{x}}-B_{\mathrm{y}}$ plane (hash marks denote $30^{\circ}$ intervals).

MPB. Within the sheath, variability is strongest near the subsolar point and decreases with increasing solar zenith angle. The upstream region is magnetically "quiet," as is the tail region inside the boundary layer or mantle. Figure $3 \mathrm{~b}$ shows variability on $30 \mathrm{~s}$ timescales; the results are robust on timescales up to $5 \mathrm{~min}$ and possibly longer.

[13] Crider et al. [2001] performed a statistical study of the draping of the IMF inside and outside of the Martian
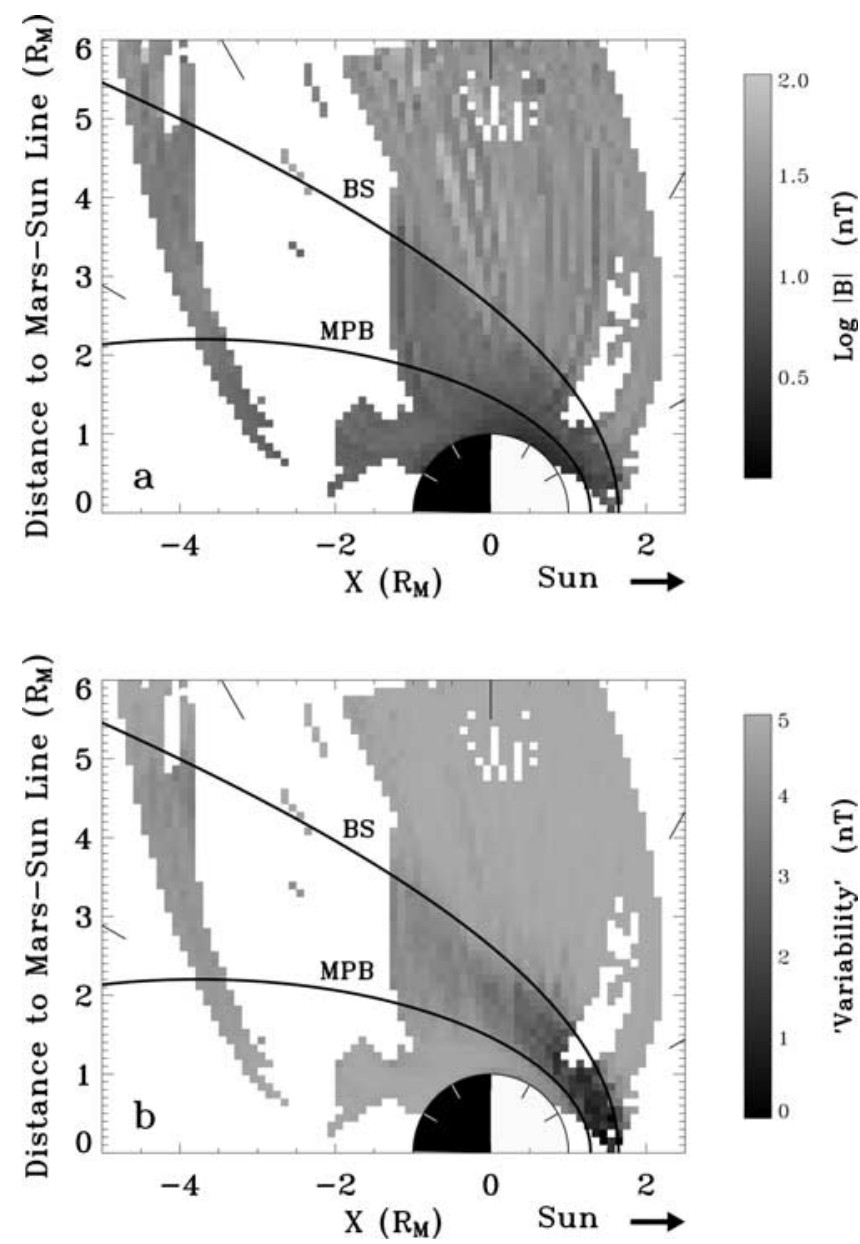

Figure 3. (a) Magnetic field magnitude and b) Linear filtered RMS as a function of location in Sun-state cylindrical coordinates. Premapping data were resampled at a $3 \mathrm{~s}$ data rate and averaged together in $30 \mathrm{~s}$ intervals. The median value in each $0.1 \times 0.1 \mathrm{R}_{\mathrm{M}}$ bin is displayed. Bins with fewer than 25 observations were not used. Independent fits to the bow shock and MPB are shown as solid lines. 


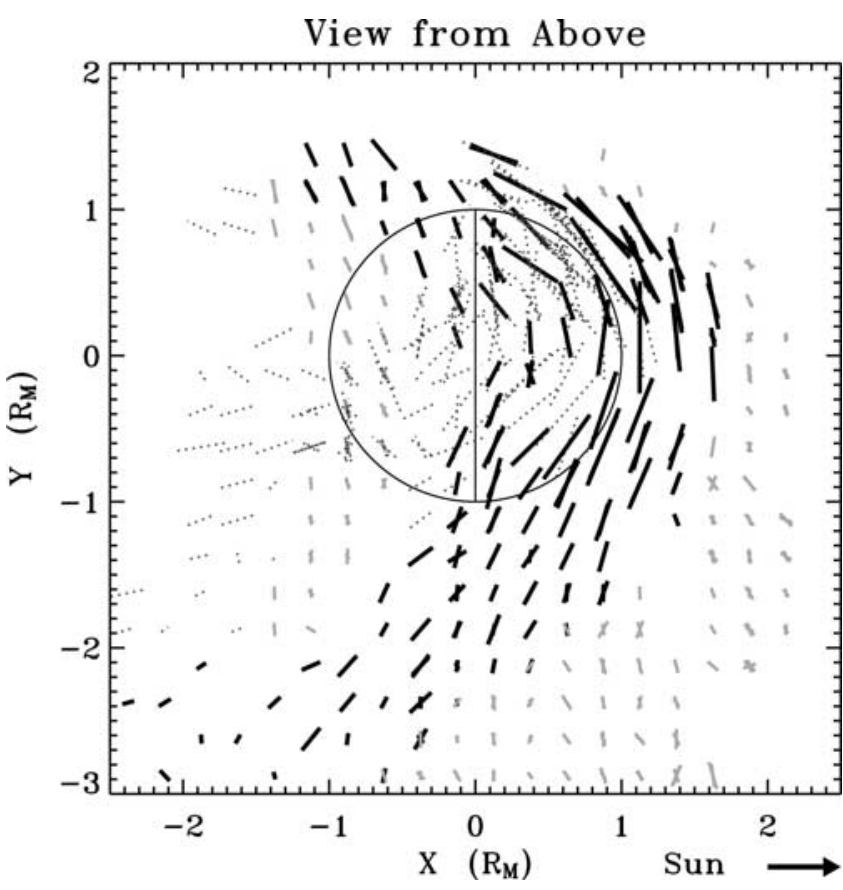

Figure 4. A projection of magnetic field vectors with altitudes less than $4 R_{M}$ onto the $x-y$ plane in Sun-state coordinates. The median vector in each bin is shown at a scale of $20 \mathrm{nT}$ per $1 \mathrm{R}_{\mathrm{M}}$, after all vectors are corrected for a sign ambiguity by flipping them so that their y component is positive. Vectors are identified according to whether they are outside the shock (light gray solid lines), between the shock and the MPB (black solid lines), or below the MPB (gray dotted lines). Since the figure is a projection of threedimensional binned data, it is possible that multiple vectors (representing bins with different distances out of the projection plane) appear in each $\mathrm{x}-\mathrm{y}$ bin.

postshock field magnitude increases, and the field drapes around the Martian obstacle, becoming mostly horizontal with respect to the planetary surface. Draping is more pronounced at low altitudes than at high altitudes. Furthermore, the field has a larger radial component at high solar zenith angles than near the sub-solar point, meaning that the shock and the MPB should be found at higher altitudes near the Martian terminator. The tail region on the Martian nightside contains field oriented mostly sunward (or antisunward); the figure shows a two-lobed structure.

\section{Morphology of Crustal Magnetic Sources}

[14] Acuña et al. [1998] first reported localized crustal magnetic sources at Mars using premapping data, and the sources were subsequently described at the MGS mapping altitude by Acuña et al. [2001]. Connerney et al. [2001] published maps of the crustal contribution to the Martian magnetic environment, and efforts to extrapolate MAG measurements down to the surface have resulted in local magnetization models [Connerney et al., 1999; Hood and Zakharian, 2001] and two published global models [Purucker et al., 2000; Arkani-Hamed, 2001]. We give a full-vector representation of the median field calculated by Connerney et al. [2001] at the mapping altitude of the spacecraft in Figure 5. As Connerney et al. [2001] showed, magnetic sources are strongest and most prevalent in the South, but crustal sources are observed at northern latitudes as well. The measured magnetic field magnitude at $\sim 100 \mathrm{~km}$ can exceed $1500 \mathrm{nT}$ over the strongest southern sources, with an estimated total net magnetic moment of $\sim 1.3 \times 10^{17} \mathrm{~A}-\mathrm{m}^{2}$ [Acuña et al., 1999].

[15] Several aspects of the nature of the Martian crustal magnetic field are apparent from the representation in Figure 5 that are not obvious in other representations. First, all three vector field components are well organized above strong crustal sources, whereas the horizontal field components above other regions of the surface are not similar in direction between adjacent bins. Therefore external fields exert (on average) a smaller fractional influence on the horizontal field component above strong crustal sources than elsewhere. The connectivity of field lines near crustal sources is also evident, with regions of positive radial field occurring near regions of negative radial field; it is possible to determine which geographic regions (at the spacecraft mapping altitude) are magnetically connected. Related to the issue of field line connectivity is the net divergence of the magnetic field calculated from the data. Both of these issues are addressed by Krymskii et al. [2002], who calculated a negative net divergence from the map published by Connerney et al. [2001] corresponding to an average radial field component of $-1.635 \mathrm{nT}$. Using the subset of mapping data described section 2, we estimate the uncertainty in this calculation at less than $0.03 \mathrm{nT}$, indicating that the Krymskii et al. [2002] result is significant. However, the uncertainty in $B_{\mathrm{r}}$ for each individual bin is much larger (on the order of $3 \mathrm{nT}$ ). This uncertainty means that the unambiguous identification of weak crustal sources at mapping altitude is difficult without more observations. For example, MAG observes weak positive radial fields in most of the bins above the Hellas impact basin (near $70 \mathrm{E}$ and $50 \mathrm{~S}$ ) but not above the Argyre impact basin (near $260 \mathrm{E}$ and $45 \mathrm{~S}$ ). However, the uncertainty in $B_{\mathrm{r}}$ for each bin, coupled with the lack of coherence of the horizontal field components from one bin to the next prevents the conclusion that crustal magnetic sources exist near Hellas from MAG data alone. Similarly, a weak hemispherical asymmetry exists in the radial field component shown in Figure 5. This asymmetry is not significant when the uncertainty in each radial field component is considered and is likely attributable to seasonal effects rather than a weak global dipole field.

[16] The strengths of many of these sources (inferred nonuniquely in the form of volume magnetization using the model presented in the work of Connerney et al. [1999]) are large enough that the crust should affect magnetic field measurements to high altitude, especially in the Southern Hemisphere. As an example, we consider the $1500 \mathrm{nT}$ observation at $100 \mathrm{~km}$ discussed above. If we assume that crustal field strength falls off with altitude as $1 / r^{3}$ (like a single dipole source) and that the source lies somewhere between the surface and $100 \mathrm{~km}$ depth [see Nimmo and Stevenson, 2001], then the crust will contribute 30 nT to the field magnitude (comparable to the compressed solar wind field of 20-40 nT) somewhere between $370 \mathrm{~km}$ and $740 \mathrm{~km}$. If instead we assume a $1 / r^{2}$ falloff (like an infinite line of dipoles or a uniformly magnetized infinite cylinder), then the altitude range is revised upward to between $700 \mathrm{~km}$ 


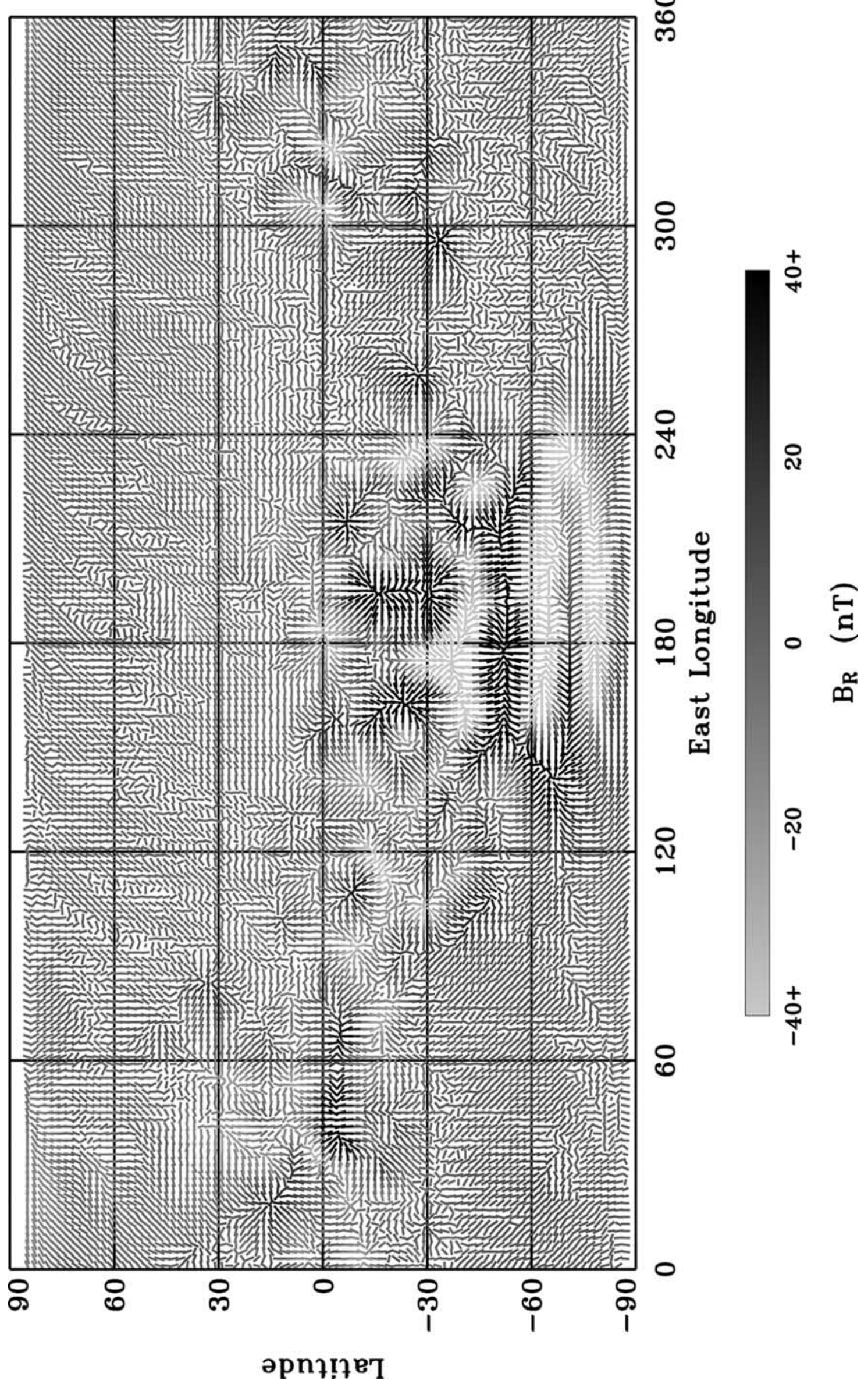


and $1400 \mathrm{~km}$. Thus we should observe large sources on Mars to altitudes as high as $\sim 1000 \mathrm{~km}$, and planetary rotation will affect magnetic field measurements at a given spacecraft position.

\section{Combined Morphology of Solar Wind and Crustal Sources}

[17] The previous two sections discussed the separate magnetic field morphologies associated with the solar wind and crustal magnetic sources observed in the averaged MAG data set. It is apparent that crustal sources complicate the draped interplanetary magnetic field topology to considerable altitudes above certain regions on the surface. The field line configuration at Mars, then, depends upon the interplanetary magnetic field strength and orientation, as well as the orientation of Mars with respect to the solar wind flow. Variability in the magnetic field structure occurs not only on time scales associated with changes in the IMF, but also on timescales associated with planetary rotation. In this section we use magnetometer data to determine how the crust and solar wind affect magnetic field observations as a function of altitude, solar zenith angle, and planetary location.

[18] We used mapping data to isolate the effects of geographic location at a constant altitude. The data were further separated according to whether or not MGS was in Mars' shadow. Figure 6 shows the contrast between observations obtained on the planetary dayside and those obtained in shadow. At $400 \mathrm{~km}$ in shadow the horizontal component of the magnetic field is low $(<10 \mathrm{nT})$, except over regions containing strong crustal sources. Dayside observations vary much more, as individual spacecraft orbits influence the average horizontal field in each longitude/latitude bin. Only the strongest crustal sources can be unambiguously distinguished on the dayside. The large difference between the Martian dayside and nightside is attributable to the draping of the interplanetary magnetic field around the conducting ionosphere. The draped field lines predominantly affect the horizontal component of the magnetic field with respect to the planetary surface; the difference between the dayside and shadow observations is less extreme for the average radial field component.

[19] Figure 6 shows that the dayside magnitude and variability of $B_{\mathrm{H}}$ are largest at midlatitudes. Midlatitudes correspond to low solar zenith angles, where the interplanetary magnetic field is most compressed and field strengths are higher, as shown in Figure 3. In Figure 7, the profile of $B_{\mathrm{H}}$ and $\left|B_{\mathrm{r}}\right|$ are plotted as a function of solar zenith angle. We minimized the effects of crustal sources by filtering out observations made above regions where the average field magnitude exceeded $10 \mathrm{nT}$ while MGS was in shadow. The horizontal field magnitude is larger than the radial field magnitude, especially in sunlight, and decreases as solar zenith angle increases. MGS passes from sunlight into shadow near $\mathrm{SZA}=120^{\circ}$, and the horizontal field decreases abruptly. By contrast, the radial field magnitude does not vary significantly with solar zenith angle.

[20] To explore the effect of altitude on the relative contributions of solar wind and crust, it is necessary to use pre-mapping data (corresponding to elliptical orbits). Figure 8 shows median altitude profiles of all premapping data below $2000 \mathrm{~km}$, partitioned according to solar zenith angle. The figure demonstrates several trends. First, the radial field component is consistently small at high altitudes and is larger and more variable at low altitudes (lower than $500-600 \mathrm{~km})$. There is little change in the radial profile with solar zenith angle. Second, each of the horizontal field components is larger and more variable than the radial field component at high altitudes on the dayside and is comparable to the radial field at low altitudes, regardless of solar zenith angle. The peak magnitude for the horizontal field components occurs below the MPB, at altitudes between 200 and $1000 \mathrm{~km}$. The size and variability of the horizontal field components decrease with increasing solar zenith angle until they match the radial profile in Mars' shadow (recall from Figure 7 that MGS was in shadow at $400 \mathrm{~km}$ when SZA $>120^{\circ}$ ). Finally, observe that $B_{\mathrm{EW}}$ (the east-west field component) tends to be larger than $B_{\mathrm{NS}}$ (the northsouth field component) in sunlight at both medium and high altitudes. The altitude at which $B_{\mathrm{EW}}$ and $B_{\mathrm{NS}}$ become comparable decreases with increasing solar zenith angle in sunlight. Each of the above trends agrees with the picture of a draped interplanetary magnetic field perturbed by the presence of crustal magnetic sources. Note that in this picture the radial field component is only weakly influenced by the IMF, with the result that the radial profile has the largest magnitude and variability at the lowest altitudes (where the influence of crustal sources is greatest). The draped interplanetary magnetic field contributes to the two horizontal field components, making them larger and more variable than the radial field component. In addition, $B_{\mathrm{EW}}$ usually exceeds $B_{\mathrm{NS}}$ because the unperturbed interplanetary magnetic field typically lies within $30^{\circ}$ of the Martian orbital plane (roughly parallel to the east-west direction), as discussed in section 3. The variability in all field components at low altitudes can be attributed to the fact that the type of crustal magnetic field (strong crustal source, weak crustal source, no crustal source) that MGS observes at low altitudes depends upon the geographic location of the orbit above the Martian surface. Nonuniform orbital coverage within each solar zenith angle range complicates many of the observed trends.

[21] The altitude to which an individual source can be discerned from the "noise" of the solar wind magnetic field depends upon many factors: the magnetization of the crust, the volume of magnetized material, the distribution of magnetized material, and (in an average sense) the depth of the material. We used premapping data to look at the radial magnetic field component (the component least

Figure 5. (opposite) The magnetic field at mapping altitude in grayscale, binned $2^{\circ} \times 2^{\circ}$ (from Connerney et al. [2001]). The full color version of this figure is binned $1^{\circ} \times 1^{\circ}$. Each vector is colored according to the radial field component, and the horizontal component is represented by the direction of each vector and the length, which is proportional to the $\log$ of the magnitude of the horizontal component $\left(.1 \mathrm{nT}=1^{\circ}\right)$. The median field vector in each bin is shown. See color version of this figure at back of this issue. 

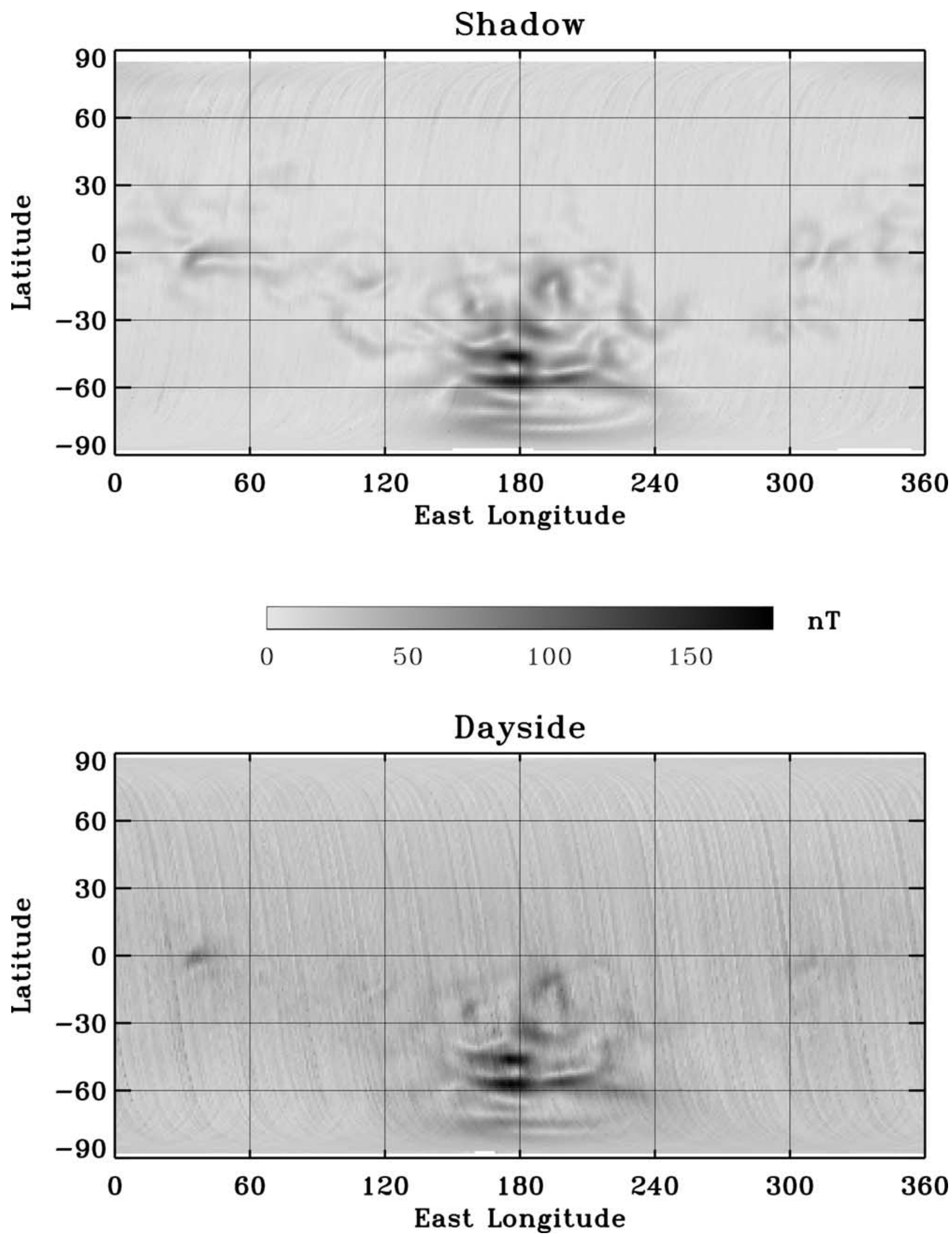

Figure 6. The average horizontal field magnitude as a function of latitude and longitude from mapping data. The top panel shows observations taken in Mars' shadow, and the bottom panel shows observations taken on the planetary dayside. $1^{\circ}$ bins are used. 


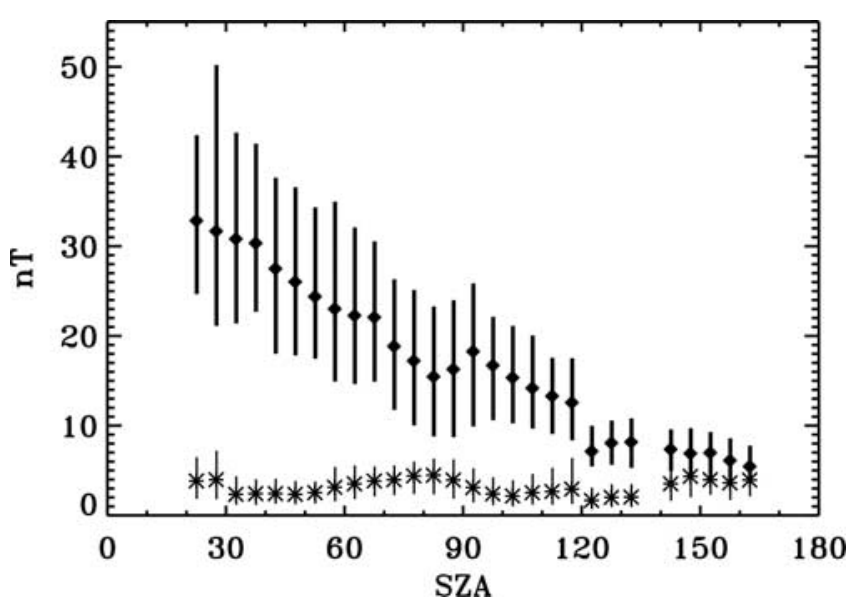

Figure 7. The median horizontal field magnitude (diamonds) at mapping altitudes is shown as a function of solar zenith angle, along with radial field magnitude (stars). Error bars denote quartiles around the median. Crustal effects are minimized by only including data from $1^{\circ}$ longitude/latitude bins where the average field magnitude is less than $10 \mathrm{nT}$ when MGS was in the planetary shadow.

contaminated by the solar wind) in three different altitude ranges (Figure 9). Owing to the sparse latitude and longitude coverage, data from all solar zenith angles are coarsely binned and averaged in $5^{\circ} \times 5^{\circ}$ bins. At low altitudes $(100-$ $200 \mathrm{~km}$ ), crustal sources are apparent over a large fraction of the planet, especially in the southern hemisphere. At altitudes from $400-500 \mathrm{~km}$, crustal sources are easily distinguished, however many of the weak sources are not distinguishable from the background field strength because they are either smaller or weaker. We are (on average) able to discern the radial field from only the large region of strong magnetization in the southern hemisphere near $180 \mathrm{E}$ at altitudes from 800 to $900 \mathrm{~km}$.

[22] The observed field magnitude at different heights above a single crustal source should yield information about the altitude to which the source effects can be observed, as well as information about the source itself. Figure 10 shows the altitude profile above a region containing the strongest southern crustal source. The field decreases rapidly up to a transition altitude near $1300-1400 \mathrm{~km}$ and falls off less steeply at higher altitudes. Above the transition altitude MAG records magnetic fields associated almost entirely with the solar wind. Similarly, the signature of crustal magnetic sources is very clear below the transition altitude. We note again that the geographic size of the region in Figure 10 is necessarily large because of the sparse premapping data coverage.

[23] A number of physical parameters should affect the field morphology above a crustal source, including the strength of the magnetization, the depth of the source, and the distribution of the magnetized material. While the actual distribution and strength of magnetized material is impossible to determine without further measurements and sophisticated modeling, we can gain insight into how the parameters above are reflected in the altitude profile of magnetic field above a given geographic region. We compare the data below $750 \mathrm{~km}$ in Figure 10 to a simple expression that incorporates each of these parameters in some form:

$$
B(z)=C\left(\frac{z+z_{0}}{z_{0}}\right)^{a}
$$
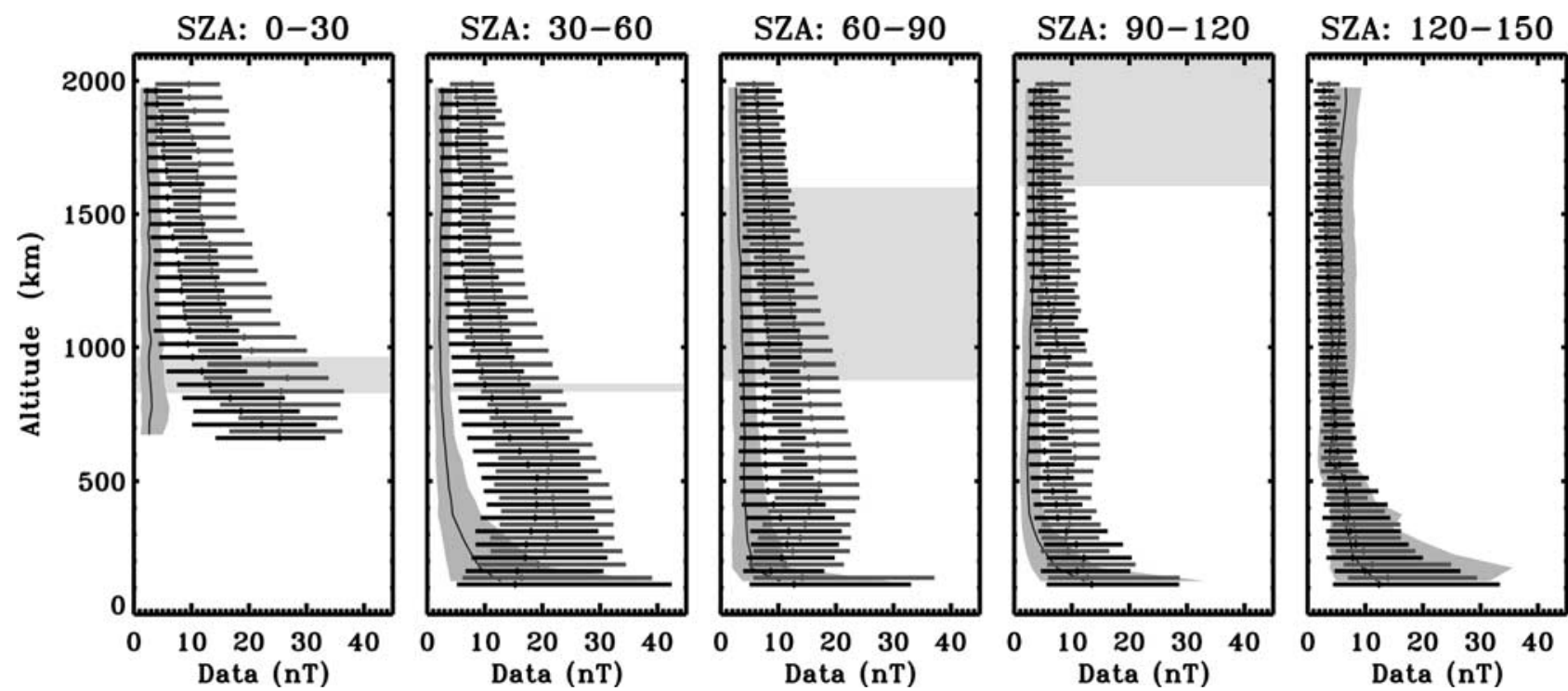

Figure 8. Median altitude profiles of premapping data, sorted according to solar zenith angle. Quartile values in each $50 \mathrm{~km}$ altitude bin up to $2000 \mathrm{~km}$ are used to estimate variability. The east-west (dark gray) and north-south (black) field component profiles are indicated by diamonds (median value) and horizontal bars (variability). The radial field component profile is indicated by the thin solid line (median value) and the light gray shaded region (variability). Altitude bins containing fewer than 200 observations are not shown. The altitude range for the best-fit MPB determined by Vignes et al. [2000] is shaded across the width of each panel. 

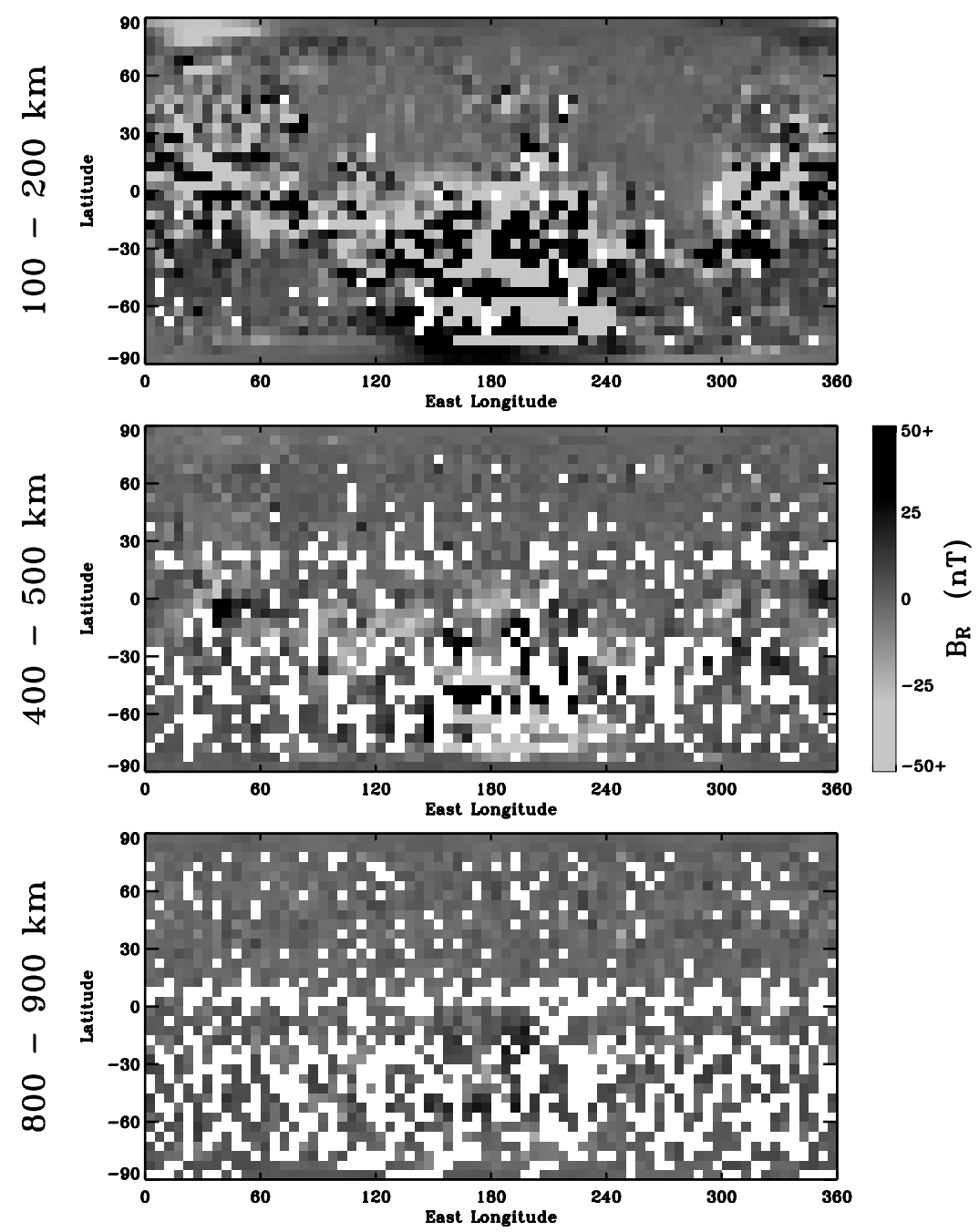

Figure 9. The median radial field component as a function of latitude and longitude, shown for three different altitude ranges. Bins are $5^{\circ} \times 5^{\circ}$. Bins with fewer than 5 observations are not shown. See color version of this figure at back of this issue.

where $z$ is altitude above the surface, $z_{0}$ is the depth of the source, $C$ is the field magnitude at the surface (and contains information about the strength of the magnetic material), and $a$ is a power law exponent (which is affected by the distribution of magnetic material). Nimmo and Stevenson [2001] constrain the depth of crustal magnetic sources to less than $100 \mathrm{~km}$, and Connerney et al. [1999] found that source depths close to $40 \mathrm{~km}$ adequately reproduced the observations. We constrain $z_{0}$ to a value of $40 \mathrm{~km}$ and perform a grid search over the strength and power law exponent parameters to determine the best fit to the observations. The best fit ( $a=$ $-2.0, C=15,800 \mathrm{nT}$ ) is shown in Figure 10 as the curve labeled " 1 ." This result for $a$ agrees with the assumption in the work of Connerney et al. [1999] that the strong southern crustal sources could be modeled by a series of infinitely long uniformly magnetized cylinders (which have a theoretical power law exponent of -2 ). We emphasize that the field strength at the surface $(C)$ and the power law exponent $(a)$ fit the data for our choice of source depth in curve "1." Different choices of source depth yield very different (and equally viable) sets of parameters. Additionally, equation (1) makes the simplifying assumption that the complex distribution of magnetization in the Martian crust can be approximated by a single source.

[24] In order to understand how the characteristics of magnetized material affect the altitude profile in Figure 10, we explore the sensitivity of equation (1) to each of the three parameters (shown in curves " 2 ," " 3 ," and " 4 "). Curve " 2 " shows the result when we increase the source depth from $40 \mathrm{~km}$ to $100 \mathrm{~km}$ (and increase $C$ by a factor of $(100 / 40)^{a}$ so that $B(z)$ remains the same at large altitudes). The power law exponent is changed to -2.2 in curve " 3 ," and $C$ is increased to $19,900 \mathrm{nT}$ in curve " 4 ." These three parameters affect the shape of the altitude profile in different ways. Increasing or decreasing $C$ shifts the profile up or down. Changing $a$ to a larger negative number (more like a dipole) makes the profile more horizontal at high altitudes. Increasing $z_{0}$ "bends" the lower altitude portion of the profile toward the vertical. The fact that the observed altitude profile "bends" toward the vertical indicates that the magnetized material is located at depth in the Martian crust.

[25] One morphological feature seen in the data in Figure 10 that is not reflected in equation (1) is the large 


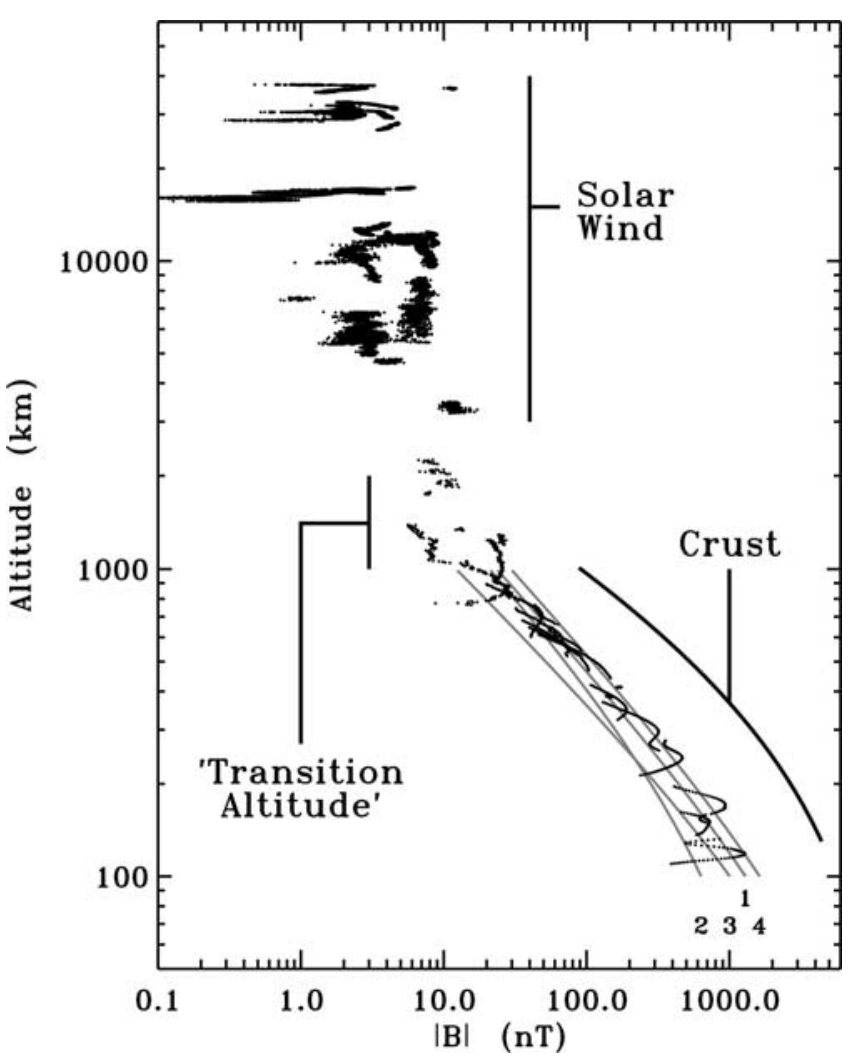

Figure 10. Field magnitude as a function of altitude for all premapping observations above the strongest Southern crustal source (170-180 E by 50-60 S). High-altitude observations primarily measure the solar wind, and lowaltitude observations primarily measure crustal magnetic fields. The numbered curves are discussed in the text.

variability in the field strengths over small altitude ranges. This variation comes from two sources. First, the altitude profile was extracted over a large region of the surface $\left(10^{\circ}\right.$ by $\left.10^{\circ}\right)$, and a single magnetized source produces a range of field magnitudes at a constant altitude over such a large area. Second, nearby crustal magnetic sources (not accounted for in our simplistic expression) contribute to the observations. These sources may be located beneath the regions of the surface we selected, and in crust outside this region. In the latter case, the relative contribution of nearby crustal sources will increase with altitude. Our selection of the region of the crust beneath the strongest observed field magnitudes likely reduces this effect over other regions we might have chosen.

[26] In summary, the technique of examining altitude profiles in MAG observations might be applied at other planetary locations (with varying levels of sophistication) given better data coverage at low altitudes. However, the sparseness of the data and the lower transition altitude between the solar wind and crust over sources other than the one studied in Figure 10 makes even qualitative fits to the data problematic.

[27] While it is impossible to uniquely extrapolate MAG data downward to determine the magnetization of the Martian crust [see, e.g., Connerney et al., 1999], we are able to estimate the vertical influence of crustal sources. Altitude profiles of premapping data analogous to the one shown in Figure 10 were analyzed over the entire planetary surface at a resolution of $10^{\circ} \times 10^{\circ}$. We determine the transition altitude for each region by looking at changes in the slope of the field magnitude profile and the profile of $B_{\mathrm{r}}$. The uncertainty in this determination is large $(\sim 100-200 \mathrm{~km})$ because of sparse data coverage and difficulties in recognizing crustal contributions (especially at high altitude). Further, because of the sparse data coverage, we use all premapping observations in our analysis regardless of solar zenith angle. However, we showed in Figure 8 that the altitude profile of the solar wind magnetic field varies with solar zenith angle, adding additional uncertainty in the identification of the transition altitude. Figure 11 shows the estimated transition altitude as a function of longitude and latitude. Fields not associated with the crust dominate observations above the indicated surface. The highest transition altitude of $1400 \mathrm{~km}$ occurs above the strongest southern source. The figure demonstrates that the Martian solar wind obstacle is not smooth; Martian crustal magnetic fields regionally contribute magnetic pressure that helps to stand off the solar wind well above the ionopause. Also, crustal sources rotate with Mars. The obstacle above each source will compress near subsolar longitudes where the component of the solar wind pressure normal to the obstacle surface is highest. Finally, the obstacle shape will fluctuate in and out as the solar wind dynamic pressure varies.

[28] Another implication of the interaction of crustal magnetic fields with the solar wind is the reconnection of magnetic field lines associated with the crust to the passing interplanetary magnetic field, providing conduits for charged particle deposition and atmospheric escape. Nightside electron measurements suggest that reconnection does occur at Mars [Mitchell et al., 2001], and Krymskii et al. [2002] report indirect topological evidence from MAG data. A full analysis of the frequency and location of magnetic field reconnection at Mars will be reported in a future paper. Here we give initial evidence from MAG data that the external magnetic field at Mars controls the field orientation above some crustal sources. Using mapping data we extracted two orbit tracks on the Martian dayside that pass above nearly identical regions on the surface. The two orbits occurred 11 months apart. Figure 12 shows the full magnetic field vector measurement projected in longitude and latitude along the orbit track for both orbits. We colored the projected vectors according to the radial component of the measured field. The third panel in Figure 12 shows the average MAG measurement at each point along the orbit track, interpolated from the Connerney et al. [2001] map. No detectable crustal magnetic sources lie below the northern part of the orbit, so the observations north of $10^{\circ}$ for both orbits are of the draped interplanetary magnetic field. Note that on 2 September the external field pointed west, while on 3 November it pointed east. The southern portion of the orbit passed over strong crustal sources. The radial component of the field is similar on both 2 September and 3 November, but the horizontal field component is quite different in the latitude range of $10-50^{\circ} \mathrm{S}$. The solar wind, then, has some control over the topology of the dayside magnetic field above crustal sources. This is even true 


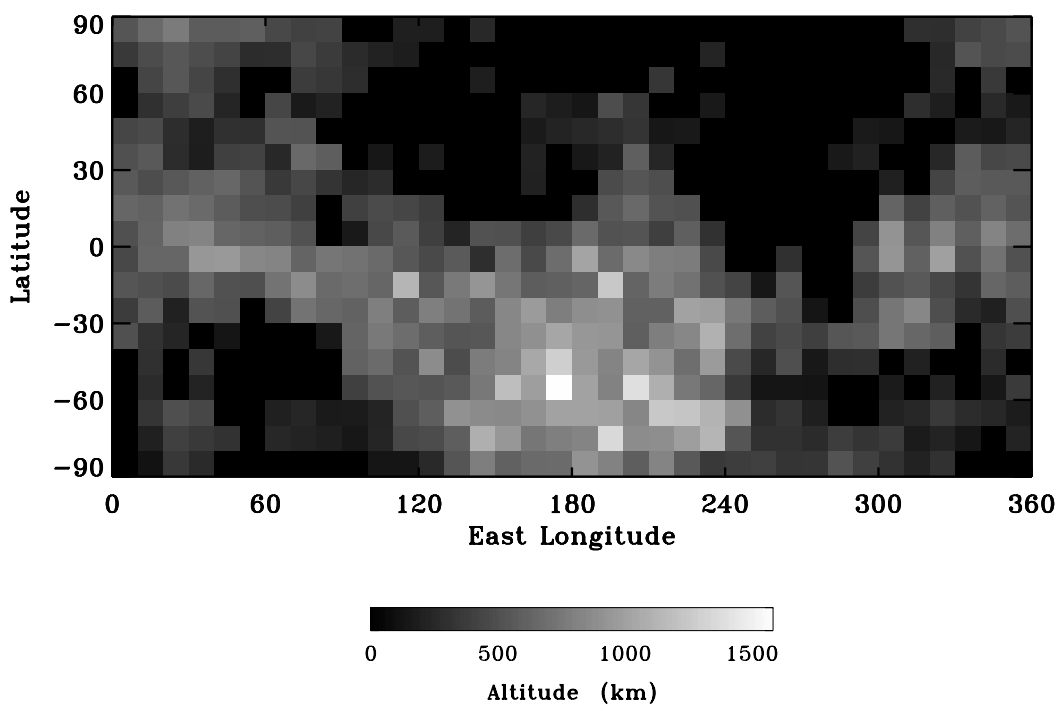

Figure 11. The altitude to which crustal magnetic fields influence MAG observations as a function of longitude and latitude is represented by identifying the "transition altitude" in each $10^{\circ}$ by $10^{\circ}$ column of premapping data.

above regions where the field magnitude from the crustal sources exceeds $80 \mathrm{nT}$.

\section{Summary}

[29] We explored magnetic morphology at Mars by analyzing MGS MAG data. The MGS mapping orbit allowed repeated observations above the entire surface at an altitude of $400 \mathrm{~km}$, and premapping orbits offered unprecedented coverage in solar zenith angle and altitude. The data coverage permitted us to isolate the effects of the solar wind and crust in certain regions and to explore the interaction of the two effects. The main findings of this work are listed below.

[30] 1. The IMF upstream from Mars is typically 2-3 nT with a spiral angle of $32^{\circ}-70^{\circ}$ and usually lies within $30^{\circ}$ of the ecliptic. The presence of spacecraft fields in MAG data (removed by Acuña et al. [2001]) limits the accuracy of the determination of the upstream field direction.

[31] 2. On average, the magnetic field increases postshock and again downstream from the MPB, has high variability between the shock and the MPB, drapes around the planet on the dayside, and exhibits a two-lobed tail structure.
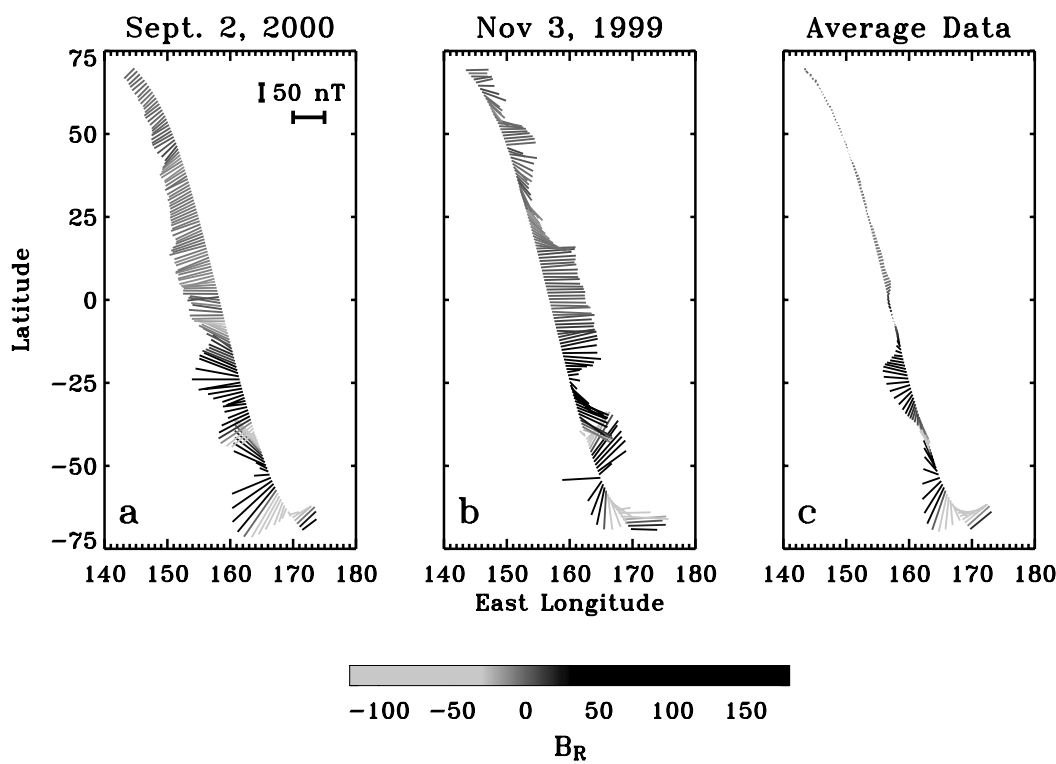

Figure 12. MAG mapping observations for two nearly identical orbit tracks: (a) 2 September 2000 and (b) 3 November 1999 . The magnetic field vector parallel to the planetary surface $\left(B_{\mathrm{H}}\right)$ is shown along each orbit track, colored according to the radial field component. The median magnetic field map calculated by Connerney et al. [2001] (and shown in Figure 5) was interpolated to the orbit track in Figure $12 \mathrm{~b}$ and is given in Figure 12c. See color version of this figure at back of this issue. 
[32] 3. Maps of crustal magnetic sources at $400 \mathrm{~km}$ altitudes show that regions of oppositely directed magnetic field are magnetically connected.

[33] 4. The magnetic field on the Martian dayside and nightside are very different at $400 \mathrm{~km}$ altitudes. The draping of the IMF affects dayside observations, making the horizontal field component larger and more turbulent than on the nightside, especially at low latitudes (nearest the subsolar point). Weak crustal sources evident in nightside data can not be distinguished on the dayside.

[34] 5. Altitude profiles of the vector magnetic field components vary with solar zenith angle, especially at altitudes higher than $250-300 \mathrm{~km}$. The IMF dominates at high altitudes and low solar zenith angles, making the horizontal field components larger and more variable than the radial field. These trends are mitigated as solar zenith angle increases. The crust dominates at altitudes lower than $200 \mathrm{~km}$, and the radial and horizontal field components (and variability) are comparable. The horizontal field magnitudes peak below the MPB.

[35] 6. The altitude to which crustal magnetic field sources influence observations varies with location. The strongest crustal source is detectable to altitudes of 1300$1400 \mathrm{~km}$. The Martian pressure balance obstacle to the solar wind occurs at different altitudes above different regions, and varies with solar wind pressure and with planetary position with respect to the solar wind.

[36] 7. Crustal field lines reconnected to the draped IMF (reported on the nightside from electron measurements by Mitchell et al. [2001]) have an undetermined geometry. However, likely candidates for reconnection include regions where the crustal field has a large radial component and the field changes direction with the IMF, analogous to polar cusps at Earth. Such regions exist at Mars in the southern hemisphere (and possibly elsewhere). In these locations the radial field component is large above strong crustal sources, and the interplanetary magnetic field drapes around the highly conducting ionosphere, contributing sufficiently to the horizontal field to change its direction.

[37] Future work will expand on the results presented here. A better understanding of the magnetic field morphology resulting from the interaction of crustal magnetic sources and the solar wind will come through comparison of the data to different models. At this point no published models include contributions from both the solar wind and crustal sources. Including both of these magnetic field contributions in a selfconsistent manner that includes the many physical effects that result from this interaction (e.g., current systems and their associated magnetic fields) is problematic. Future models for the Martian field topology should be validated by MAG data. An accurate understanding of the magnetic morphology at Mars will allow us to describe the shape and variability of the Martian solar wind obstacle for comparison with observations by future spacecraft. Furthermore, it will enable prediction of the motion of charged particles along field lines near Mars, which is an important component in the understanding of atmospheric loss to the solar wind and particle deposition into the Martian ionosphere.

[38] Acknowledgments. D. Brain would like to thank the MGS MAG/ER Team, Pat Lawton, and Monte Kaelberer. He acknowledges useful conversations with D.H. Crider and J.G. Luhmann. This work was supported by NASA Grant NAG5-11177.
[39] Lou-Chuang Lee thanks Jafar Arkani-Hamed for the assistance in evaluating this paper.

\section{References}

Acuña, M. H., et al., The magnetic field of Mars: Summary of results from the aerobraking and mapping orbits, J. Geophys. Res., 106, 23,40323,418, 2001.

Acuña, M. H., et al., Global distribution of crustal magnetization discovered by the Mars Global Surveyor MAG/ER experiment, Science, 284, 790793, 1999.

Acuña, M. H., et al., Magnetic field and plasma observations at Mars: Initial results from the Mars Global Surveyor Mission, Science, 279, 16761680, 1998.

Acuña, M. H., et al., Mars Observer Magnetic Fields Investigation, J. Geophys. Res., 97, 7799-7814, 1992.

Arkani-Hamed, J., A 50-degree spherical harmonic model of the magnetic field of Mars, J. Geophys. Res., 196, 23,197-23,209, 2001.

Bogdanov, A. V., and O. L. Vaisberg, Structure and variations of solar wind-Mars interaction region, J. Geophys. Res., 80, 480, 1975.

Brain, D. A., The influence of crustal magnetic sources on the topology of the Martian magnetic environment, Ph.D. thesis, Univ. of Colorado, Boulder, Colo., 2002.

Brecht, S. H., Hybrid simulations of the magnetic topology of Mars, J. Geophys. Res., 102, 4743-4750, 1997.

Breus, T. K., The solar wind interaction with Mars over the solar cycle: A post-Phobos view, in Venus and Mars: Atmospheres, Ionospheres, and Solar Wind Interactions, edited by J. G. Luhmann, M. Tatrallyay, and R. O. Pepin, pp. 387-403, AGU, Washington, D. C., 1992.

Cloutier, P. A., et al., Venus-like interaction of the solar wind with Mars, Geophys. Res. Lett., 26, 2685-2688, 1999.

Connerney, J. E. P., M. H. Acuña, P. J. Wasilewski, N. F. Ness, H. Réme, C. Mazelle, D. Vignes, R. P. Lin, D. L. Mitchell, and P. A. Cloutier, Magnetic lineations in the ancient crust of Mars, Science, 284, 794798,1999

Connerney, J. E. P., M. H. Acuña, P. J. Wasilewski, G. Kletetschka, N. F. Ness, H. Réme, R. P. Lin, and D. L. Mitchell, The global magnetic field of Mars and implications for crustal evolution, Geophys. Res. Lett., 28, 4015-4018, 2001

Crider, D., M. Acuña, J. Connerney, D. Mitchell, R. Lin, P. Cloutier, H. Rème, C. Mazelle, D. Brain, N. Ness, and S. Bauer, Magnetic field draping around Mars: Mars Global Surveyor results, Adv. Space Res., 27, 1831-1836, 2001.

Curtis, B. L., and N. F. Ness, Remanent magnetism at Mars, Geophys. Res. Lett., 15, 737-739, 1988

Dolginov, S. S., Y. G. Yeroshenko, and L. N. Zhuzgov, The magnetic field of Mars according to the data from the Mars 3 and Mars 5, J. Geophys. Res., 19, 3353-3362, 1976

Hood, L. L., and A. Zakharian, Mapping and modeling of magnetic anomalies in the northern polar region of Mars, J. Geophys. Res., 106, 14,601$14,620,2001$.

Krymskii, A. M., T. K. Breus, M. H. Acuña, J. E. P. Connerney, D. H. Crider, D. L. Mitchell, and S. J. Bauer, Structure of the magnetic field fluxes connected with crustal magnetization and top-side ionosphere at Mars, J. Geophys. Res., 107(A9), 1245, doi:10.1029/2001JA000239, 2002.

Law, C. C., and P. A. Cloutier, Observations of magnetic structure at the dayside ionopause of Venus, J. Geophys. Res., 100, 23,973-23,981, 1995.

Liu, Y., A. F. Nagy, C. P. T. Groth, D. L. DeZeeuw, and T. I. Gombosi, 3D multi-fluid MHD studies of the solar wind interaction with Mars, Icarus, 26, 2689-2692, 1999

Luhmann, J. G., C. T. Russell, L. H. Brace, and O. L. Vaisberg, The intrinsic magnetic field and solar-wind interaction of Mars, in Mars, edited by H. Kieffer et al., pp. 1090-1134, Univ. of Ariz. Press, Tucson, Ariz., 1992.

Lundin, R., A. Zakharov, R. Pellinen, H. Borg, E. M. Dubinin, B. Hultqvist, H. Koskinen, I. Liede, and N. Pissarenko, ASPERA/Phobos measurements of the ion outflow from the Martian ionosphere, Geophys. Res. Lett., 17, 873-876, 1990a.

Lundin, R., A. Zakharov, R. Pellinen, H. Borg, B. Hultqvist, N. Pissarenko, E. M. Dubinin, S. W. Barabash, I. Liede, and H. Koskinen, Plasma composition measurements of the Martian magnetosphere morphology, Geophys. Res. Lett., 17, 877-880, 1990b.

Mitchell, D. L., R. P. Lin, C. Mazelle, H. Rème, P. A. Cloutier, J. E. P. Connerney, M. H. Acuña, and N. F. Ness, Probing Mars' crustal magnetic field and ionosphere with the MGS Electron Reflectometer, J. Geophys. Res., 106, 23,419-23,427, 2001.

Nimmo, F., and D. J. Stevenson, Estimates of Martian crustal thickness from viscous relaxation of topography, J. Geophys. Res., 106, 50855098, 2001 
Purucker, M., D. Ravat, H. Frey, C. Voorhies, T. Sabaka, and M. Acua, An altitude-normalized magnetic map of Mars and its interpretation, Geophys. Res. Lett., 27, 2449-2452, 2000

Russell, C. T., et al., The magnetic field of Mars: Mars 5 evidence reexamined, Geophys. Res. Lett., 5, 81, 1978.

Schwingenschuh, K., W. Riedler, H. Lichtenegger, Y. Yeroshenko, K. Sauer, J. G. Luhmann, M. Ong, and C. T. Russell, Martian bow shock: Phobos observations, Geophys. Res. Lett., 17, 889-892, 1990.

Spreiter, J. R., and S. S. Stahara, A new predictive model for determining solar wind-terrestrial planet interactions, J. Geophys. Res., 85, 67696777, 1980.

Szego, K., Present understanding of the dayside mantle regions of Venus and Mars, Adv. Space Res., 28, 841-850, 2001.

Vaisberg, O. L., The solar wind interaction with Mars: A review of results from early soviet missions to Mars, in Venus and Mars: Atmospheres, Ionospheres, and Solar Wind Interactions, edited by J. G. Luhmann, M. Tatrallyay, and R. O. Pepin, pp. 311-326, AGU, Washington, D. C., 1992.
Vignes, D., C. Mazelle, H. Reme, M. H. Acuña, J. E. P. Connerney, R. P. Lin, D. L. Mitchell, P. Cloutier, D. H. Crider, and N. F. Ness, The Solar Wind interaction with Mars: Locations and shapes of the Bow Shock and Magnetic Pile-up Boundary from the observations of the MAG/ER experiment onboard Mars Global Surveyor, Geophys. Res. Lett., 27, 49-52, 2000

Zakharov, A. V., The plasma environment of Mars: Phobos mission results, in Venus and Mars: Atmospheres, Ionospheres, and Solar Wind Interactions, edited by J. G. Luhmann, M. Tatrallyay, and R. O. Pepin, pp. $327-$ 344, AGU, Washington, D. C., 1992.

M. H. Acuña and J. E. P. Connerney, NASA Goddard Space Flight Center, Code 695.0, Greenbelt, MD 20701, USA. (mario.acuna@gsfc. nasa.gov; john.connerney@gsfc.nasa.gov)

F. Bagenal and D. A. Brain, Laboratory for Atmospheric and Space Physics, University of Colorado, Campus Box 392, Boulder, CO 80309, USA. (bagenal@lasp.colorado.edu; david.brain@colorado.edu) 


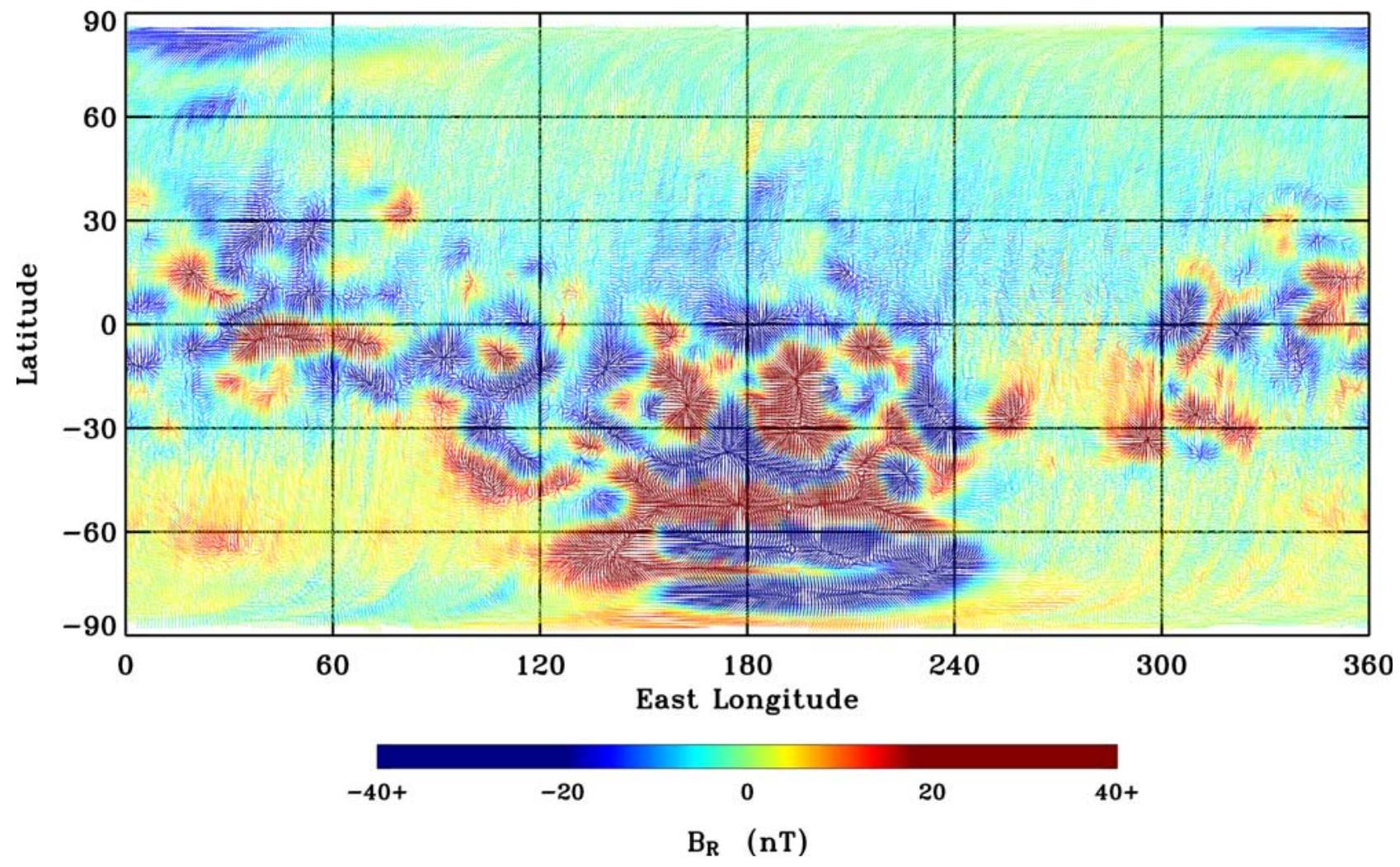

Figure 5. The magnetic field at mapping altitude in grayscale, binned $2^{\circ} \times 2^{\circ}$ (from Connerney et al. [2001]). The full color version of this figure is binned $1^{\circ} \times 1^{\circ}$. Each vector is colored according to the radial field component, and the horizontal component is represented by the direction of each vector and the length, which is proportional to the log of the magnitude of the horizontal component $\left(.1 \mathrm{nT}=1^{\circ}\right)$. The median field vector in each bin is shown. 

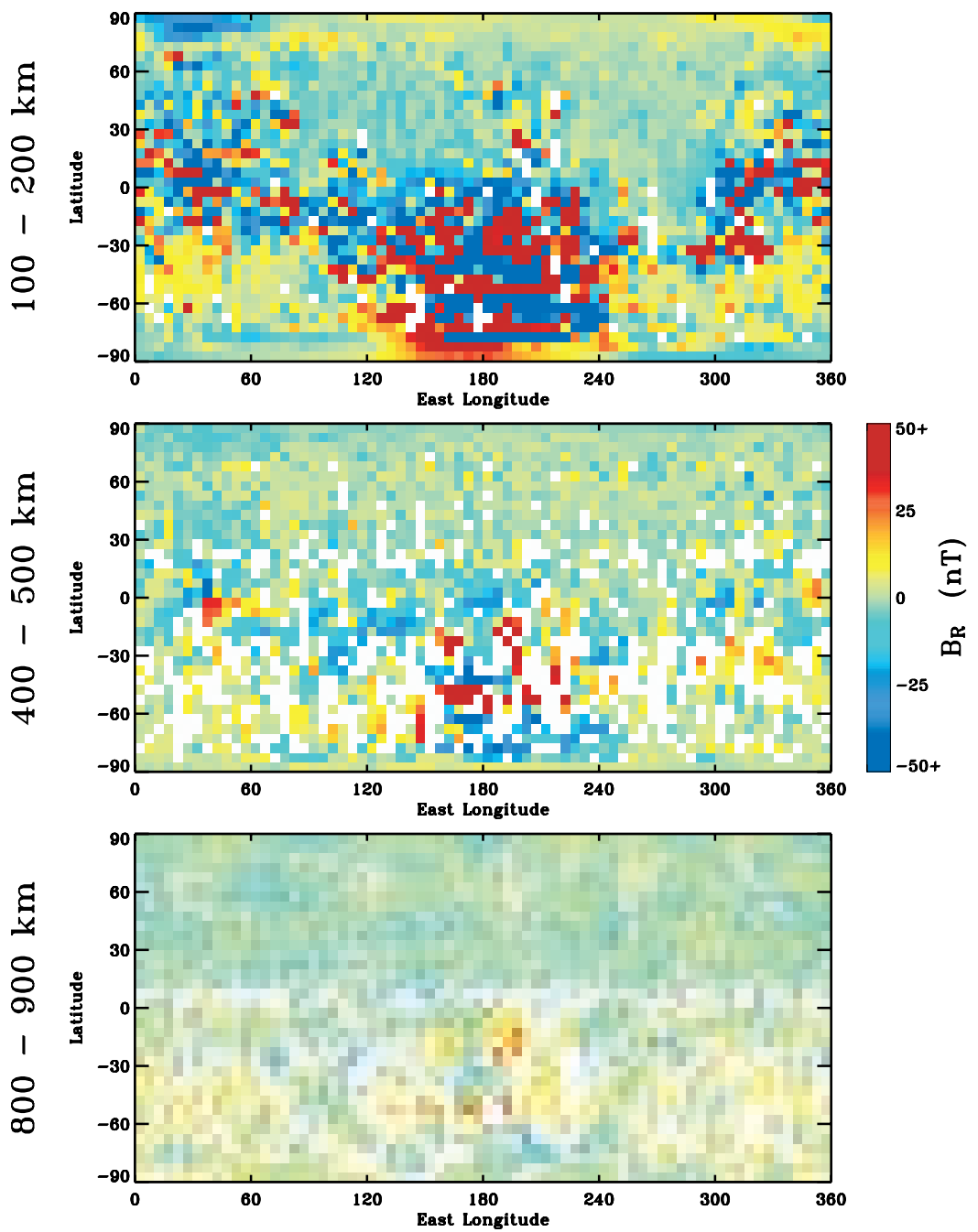

Figure 9. The median radial field component as a function of latitude and longitude, shown for three different altitude ranges. Bins are $5^{\circ} \times 5^{\circ}$. Bins with fewer than 5 observations are not shown. 

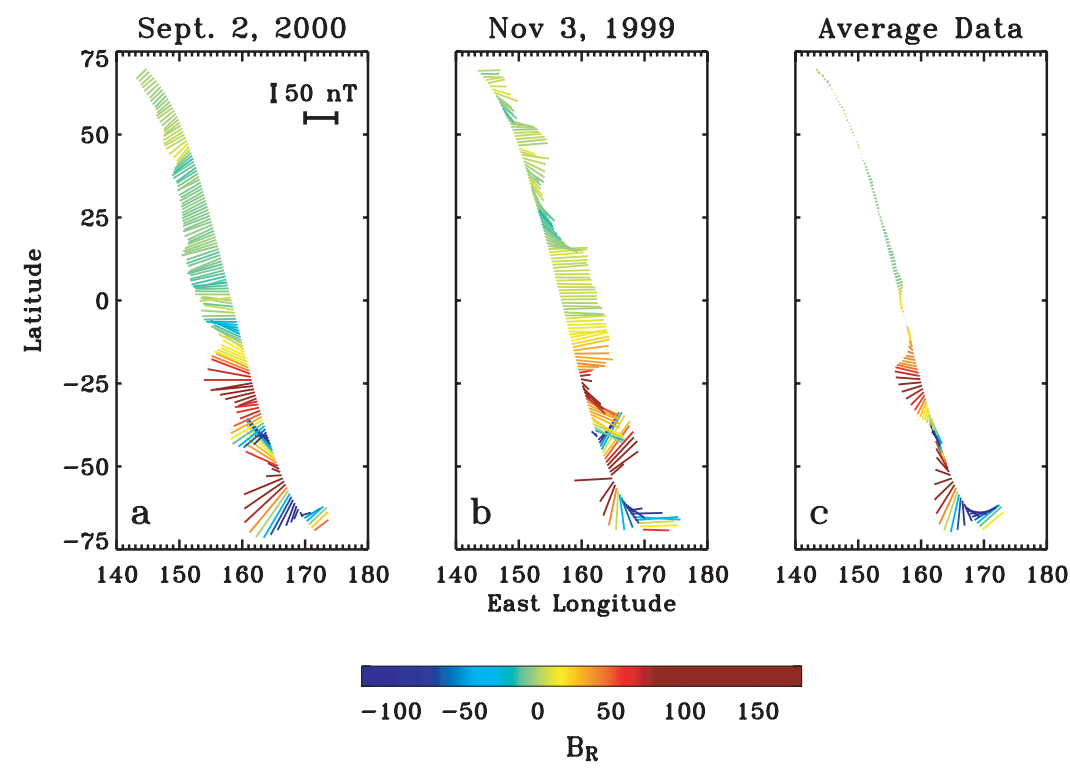

Figure 12. MAG mapping observations for two nearly identical orbit tracks: (a) 2 September 2000 and (b) 3 November 1999. The magnetic field vector parallel to the planetary surface $\left(B_{\mathrm{H}}\right)$ is shown along each orbit track, colored according to the radial field component. The median magnetic field map calculated by Connerney et al. [2001] (and shown in Figure 5) was interpolated to the orbit track in Figure $12 \mathrm{~b}$ and is given in Figure 12c. 